\title{
Difficulté de se dire. Sur l'écriture autobiographique de Françoise Mallet-Joris
}

\author{
Difficulty talking about yourself. On the \\ autobiographical writing of Françoise Mallet-Joris
}

\author{
Renata BIZEK-TATARA \\ Université Marie-Curie Skłodowska \\ r.bizek-tatara@poczta.umcs.lublin.pl
}

\begin{abstract}
This article deals with four autobiographical texts by the Belgian writer Françoise Mallet-Joris (1930-2017): Lettre à moi-même (1963), La Maison de papier (1970), J'aurais voulu jouer de l'accordéon (1975) and La double confidence (2000). These texts, despite being inspired by the writer's life experiences, are far from Philippe Lejeune's autobiographical model because the author, instead of talking about herself, narrates about others. The difficulties of speaking about her own life determines the somewhat unusual character of these texts: they are ambiguous genres that are placed between autobiography, memories, intimate diary, essay, biography and chronicle. In the analysis, I focus the author's choice of theme (literary work, life of others, biography of Marcelina Debordes-Valmore) and form (hybrid genres, mosaic structure, fragmentarism) which makes this unique "writing about oneself" so original. I also try to find out why the author is unable to write about herself and how that influences the shape of her autobiographical texts.
\end{abstract}

\begin{abstract}
Resumen
El presente artículo trata sobre cuatro textos autobiográficos de la escritora belga Françoise Mallet-Joris (1930-2017): Lettre à moi-même (1963), La Maison de papier (1970), J'aurais voulu jouer de l'accordéon (1975) y La Double confidence (2000). Estos textos, a pesar de estar inspirados en las experiencias vitales de la escritora, se alejan considerablemente del modelo autobiográfico de Philippe Lejeune porque su autora, en vez de hablar de sí misma, cuenta sobre los demás. Las dificultades de hablar de su propia vida influyen en el carácter un tanto inusual de esos textos: son géneros ambiguos que se sitúan entre autobiografía, memorias, diario íntimo, ensayo, biografía y crónica. En los análisis me centro en la temática (obra literaria, vida de los demás, biografía de Marcelina Debordes-Valmore) y en la forma (géneros híbridos, estructura de mosaico, fragmentarismo) que elige la autora y que hacen su "escritura sobre sí misma" tan original. Asimismo trato de averiguar por qué la autora se ve incapaz de escribir sobre sí misma y cómo aquello influye en la forma de sus textos autobiográficos.
\end{abstract}


Anales de Filología Francesa, n. ${ }^{\circ}$ 27, 2019

Difficulté de Se dire. Sur L'ÉCriture autobiographique de Françoise Mallet-Joris

\section{Key-words}

Belgian literature, Françoise Mallet-Joris, autobiography, gender syncretism, fragmentarism, mosaic structure.

\section{Palabras clave}

literatura belga, Françoise Mallet-Joris, autobiografía, sincretismo de géneros, fragmentarismo, estructura de mosaico.

\section{Introduction}

Aucun lecteur de Françoise Mallet-Joris (1930-2016) ne s'étonnera de voir son nom cité dans une enquête sur les écritures du moi, car toute sa production littéraire, aussi abondante et diversifiée qu'elle soit, est généreusement nourrie de son vécu. Non seulement la romancière belge s'en inspire pour tisser la trame de ses fictions (romans et nouvelles), mais elle y consacre également quatre ouvrages autobiographiques: Lettre à moi-même (1963), La Maison de papier (1970), J'aurais voulu jouer de l'accordéon (1975) et La Double confidence (2000). L'auteure exprime clairement son intérêt pour l'écriture autobiographique: “[...] me voilà ramenée au vrai, aux faux, et à moi-même. À ces questions qui me viennent à l'esprit de m'être vue, reflétée dans un miroir humain, comme une personne, et pourquoi pas, comme un personnage?" (Mallet-Joris, 1963: 80-81). Elle ajoute à un autre endroit: “J'aime raconter. [...] c'est moi tout entière qui m'exprime, dans cette histoire, dans ces images qui m'enivrent un peu" (Mallet-Joris, 1970: 204).

Toutefois, malgré cette volonté explicitement déclarée de raconter sa vie et la facilité d'en parler lors des innombrables interviews que l'écrivaine a donnés au cours de sa longue carrière, elle n'arrive pas, à tout le moins difficilement, à dévoiler son intimité et à rédiger une autobiographie comprise comme "le récit de vie centré sur l'histoire de sa personnalité" (Lejeune, 1971: 10). En effet, au lieu d'y parler d'elle-même, de son vécu, Françoise Mallet-Joris parle des autres. La place qu'elle accorde à autrui et sa réserve observée à l'égard de la confidence intime bafouent des lois fondamentales de l'autobiographie et engendrent des formes inhabituelles et difficilement classables: ses quatre récits de soi amalgament des ingrédients de l'autobiographie, des mémoires, de la confession, de l'essai, de la biographie, de la chronique et, dans La Double confidence, du récit de filiation et du récit de deuil. Dans notre propos, nous réfléchirons sur les choix thématiques et formels de l'auteure qui contribuent à la singularité de ses écritures du moi, ainsi que sur les origines de cette difficulté de se dire et l'impact de celle-ci sur sa production littéraire.

Les quatre textes en question disent "je” de manière explicite, ce qui n'est pas si

1 Françoise Mallet-Joris, nom de plume de Françoise Lilar, est née à Anvers en 1930. Elle passe son enfance en Belgique, auprès de ses parents, le ministre de la Justice Albert Lilar et l'écrivaine Suzanne Lilar, puis s'installe à Paris. Après la publication en 1951, très remarquée, de son premier ouvrage Le Rempart des Béguines, elle continue à écrire et obtient en 1958 le Prix Femina pour L'Empire céleste. Son œuvre se compose de romans, nouvelles, biographies (de Marie Mancini et de Jeanne Guyon), romans historiques et récits autobiographiques. Elle a été membre du jury Prix-Femina. En 1970, elle a été élue à l'Académie Goncourt et, en 1994, à l'Académie royale de langue et littérature françaises. 
fréquent à l'époque postmoderne qui "fait naître une autobiographie qui tourne à la pseudoautobiographie, où le 'je' n'est pas un vrai 'je', mais un simple pronom personnel, gageure de pseudovérité, un appât pour un lecteur blasé, friand du non-dit” (Rachwalska von Rejchwald, 2007: 72). Cependant, bien que l'auteure s'identifie de façon évidente au narrateur et au personnage, ses ouvrages ne s'inscrivent que partiellement dans la célèbre définition de l'autobiographie, proposée par Philippe Lejeune: c'est “le récit rétrospectif en prose qu'une personne réelle fait de sa propre existence, lorsqu'elle met l'accent sur sa vie individuelle, en particulier sur l'histoire de sa personnalité" (Lejeune, 1971: 14). Essayons donc de révéler dans quelle mesure Françoise Mallet-Joris se conforme aux règles sacro-saintes de l'autobiographie et dans quelle mesure elle les transgresse pour donner à ses récits de soi une coloration originale et fort personnelle.

\section{Aux confins de l'autobiographie et de l'essai: Lettre à moi-même et J'aurais voulu jouer de l'accordéon}

Tout lecteur de Lettre à moi-même qui chercherait à se faire admettre parmi les ayants droit aux confessions intimes sera immanquablement déçu, car il n'y trouvera pas de détails croustillants de la vie privée de l'auteure. Françoise Mallet-Joris y réfléchit sur le métier d'écrivain et sur le statut de la femme auteur, ce qui apparente visiblement son texte à une "biographie intellectuelle" (Petit, 2001: 109) ou, selon les mots de la romancière, à l'essai (Paque, 2017). Elle invite le lecteur dans son atelier d'écriture et lui révèle les secrets de son art d'écrire: comment et dans quelles conditions elle travaille, comment naissent ses textes, à quels modes et procédés de composition elle recourt, comment elle choisit les sujets, conçoit l'intrigue et construit les personnages, quels sont les traits caractéristiques de son œuvre et, enfin, dans quelle mesure elle s’identifie à son image publique, Françoise Mallet-Joris écrivain, façonnée et diffusée par la critique et les journalistes ${ }^{2}$. Quant à sa vie intime, c'est-à-dire à son image privée, celle de Françoise Mallet-Joris femme, maîtresse, mère, fille, amie, elle en livre très peu d'informations. Elle ne révèle pas l'identité de ses parents ni celle de ses maris et se tait sur sa vie familiale. Il en est de même avec ses amis qu'elle désigne par leur seul prénom, accompagné ou non d'une initiale, et gomme leurs traits les plus reconnaissables. Le choix du pseudonyme, tout au début de sa carrière d'écrivain, lui garantit l'anonymat et protège efficacement sa vie privée et celle de ses parents ${ }^{3}$ Comme beaucoup d'autres femmes écrivains éprises de l'autobiographique, telles Nathalie Sarraute ou Marguerite Yourcenar ( $c f$. Lecarme et Lecarme-Tabone, 1997), Françoise Mallet-Joris se démarque du projet confes-

2 Le thème de l'écriture de Françoise Mallet-Joris est exhaustivement étudié par Joanna Pychowska, 2007: 6570).

3 Elle signe d'un pseudonyme son premier roman Le Rempart des béguines (1951) pour épargner à ses parents les inconvénients d'un possible scandale au cas où cette histoire d'un amour homosexuel serait interprétée comme autobiographique. 
sionnel de Jean-Jacques Rousseau, trop indiscret, impudique même, dans ses aveux et dans les implications de ses proches ${ }^{4}$.

Cependant, cette réticence surprend, car dans de nombreux entretiens que Françoise Mallet-Joris a accordés à la télé et à la presse, elle parlait volontiers de sa vie quotidienne, de ses quatre enfants, de sa conversion au christianisme ou de l'écriture de sa mère, Suzanne Lilar, et donnait aisément son avis sur la réalité sociopolitique de son temps. Il s'agit donc moins de la protection de sa vie privée que de l'impossibilité d'écrire sur ce sujet. À cette époque-là, la romancière n'est pas encore prête à une telle exhibition de soi. Elle le confirme:

[...] mots abstraits, concrètes vérités, un sujet assez noble en somme. L'appréhender avec certaines précautions. Lectures préalables, cahiers pour les citations, classeur peut-être. Pas un roman en tout cas. L'histoire de ma vie jusqu'à ce jour. L'histoire de la vie et moi jusqu'à ce jour. L'histoire de mon effort pour vivre, de ma recherche de cette aisance de vivre, de cette machine de vivre... Trop difficile. Pas assez de lectures. Esprit trop vite lassé des mots abstraits, trop vite rassasié. Trop prompte à sécréter des images pour pouvoir suivre un raisonnement jusqu'au bout. Trop acharné à incarner chaque mot. Non, trop tôt pour écrire ce livre (Mallet-Joris, 1963: 29-30).

C'est pourquoi, au lieu de parler d'elle-même, elle préfère parler des autres, de ceux qu'elle connaît ou ne connaît pas: amis, collègues, écrivains, critiques, journalistes, concierges, patrons de café ou inconnus, simples passants ou clients croisés dans la rue ou au bistrot ${ }^{5}$. Ainsi, elle dresse le tableau de la comédie sociale parisienne, croque d'une plume alerte et quelquefois cinglante les jeux de rôle auxquels elle assiste ou participe: interview de journaliste, dîner d'amis, conversation avec un commerçant raciste ou rencontre avec un cinéaste.

Ajoutons que Françoise Mallet-Joris n'est pas à la recherche de l'original, de l'extraordinaire, tout au contraire, elle est visiblement fascinée par le quotidien, qu'il soit familier ou ordinaire. C'est une observatrice infatigable qui scrute le réel avec une singulière acuité du regard et saisit les facéties imprévisibles de l'existence humaine. Ce qui l'intéresse, ce n'est pas son expérience personnelle, mais l'expérience commune, propre à ses contemporains. Elle le confirme dans une interview où elle commente son geste autobiographique:

$\mathrm{Au}$ fond, je ne parle pas de moi-même, tellement. Je parle d'une expérience que j'ai faite, mais toujours, en écrivant, j'avais le sentiment d'apporter un témoignage sur une chose que j'avais vécue mais qui appartenait à tout le monde plutôt que celui de raconter une chose qui m'était propre, à moi seule (Detry, 1976: 30).

4 Elle avoue: "Rousseau m'agace énormément, et il continue à m'agacer. C'est un épanchement sans force. Je sens une espèce d'attendrissement sur soi-même qui me déplait. Je trouve que c'est complaisant" (Detry, 1976: 30).

5 Par là, Lettre à moi-même nous fait penser au journal intime La Vie extérieure (2000) d'Annie Ernaux où la romancière consigne la vie des autres et pas la sienne. Elle constate: "Je note ici les signes d'une époque, rien d'individuel" (Ernaux, 2000: 99). 
Par là, sa Lettre à moi-même s'apparente à la chronique qui se veut le récit détaillé des événements vus par un témoin privilégié. L'énonciation faite à la première personne lui donne son identité de témoignage et d'analyse, partagé entre la subjectivité de l'expérience vécue et l'objectivité du regard extérieur. Celui-ci lui sert à mieux légitimer sa vérité sur le monde, comme si la subjectivité exprimée par le récit de soi, à cause justement de son caractère personnel, intime et unique, donc invérifiable, n'était pas assez véridique et digne de confiance. Cette perspective "extérieure" pourrait jouer ici la fonction d'une caution objectivante.

Ce regard extérieur, elle le pose aussi sur elle-même et essaye de parler d'elle-même comme d'un personnage de roman. Nous lisons au seuil du livre:

Et cette Mallet-Joris paisible et assurée [...]. Je contemple cette Mallet-Joris qui fait
toujours bien en toutes saisons, dans tous les décors, pourvu qu'elle y mette un peu de
bonne volonté. Sous un arbre de Noël (C'est dommage, vous avez coupé vos cheveux, le
chignon faisait plus ... comment dire ...) Oui, comment dire ? Je la vois faisant les de-
voirs d'un de ses enfants, ou penchée au chevet de son dernier-né (Vous savez que c'est
le quatrième? [...] Et la légende? Elle a divorcé deux fois, vous savez, mais après tout,
cela prouve son goût pour le mariage, non? Non, on ne le met pas, les lectrices ...) et il
me semble que nous avons bien peu de choses en commun (Mallet-Joris, 1963: 14-15).

Un tel dédoublement (mis en évidence par l'usage des caractères différents) traduit parfaitement la difficulté à écrire sur elle-même, saisir sa personne dans sa totalité, dire la vérité sur soi et se dévoiler à autrui. L'auteure cherche à s'effacer comme productrice de son discours et à gommer délibérément toute marque personnelle pour se considérer comme une elle, Françoise Mallet-Joris écrivain. C'est une façon de repousser toute tentation de s'intéresser à sa vie intime qui serait non pertinente dans le cadre d'une autobiographie intellectuelle. La lecture de Lettre à moi-même révèle que parler de soi à la troisième personne, qui a permis à nombre d'écrivains de s'observer dans le texte comme dans le miroir, comme si moi était un autre, s'avère ici inopérant et l'auteure y renonce aussitôt: dans la suite du texte, elle assurera une communion parfaite entre le narrateur et le personnage et s'exprimera par "je".

J'aurais voulu jouer de l'accordéon ressemble à bien des égards à Lettre à moi-même et peut être considéré comme son deuxième volet. Dans ce petit livre, écrit en deux semaines pour la collection "Idée fixe" chez Julliard, Françoise Mallet-Joris continue à témoigner sur son métier d'écrivain et, en particulier, sur le processus d'écriture d'un livre. Elle y aborde la question de ses désirs, antithétiques et inconciliables, de se cacher devant le public et d'avoir une relation directe avec ses lecteurs. D'où le motif de l'accordéon dont elle aimerait jouer au lieu d'écrire et de répondre aux questions concernant sa vie privée: selon la romancière, l'accordéoniste se dissimule derrière son instrument et n'existe pour le public que par sa fonction, le temps de jouer, en conjuguant l'immédiateté et la distance (Mallet-Joris, 1975: 34-35). Avec l'écriture, c'est tout à fait différent: tout auteur accomplit une performance privée et, 
quand le livre est publié, les pensées de l'écrivain sont loin, concentrées sur le texte suivant. Qui plus est, on attend de lui d'avoir un avis sur tout: "Oh ! j'aurais voulu jouer de l'accordéon! On ne m'aurait jamais demandé mon opinion sur l'éducation des enfants", avoue-t-elle (Mallet-Joris, 1975: 34). Comme le constate justement Susan Petit, la romancière "insiste sur la fracture entre le personnage public et l'auteur solitaire, entre son impulsivité et son besoin de prudence et d'ordre, entre sa crainte de prendre position et son désir de faire part de ce qu'elle pense, entre son besoin et sa crainte de se révéler" (Petit, 2001: 126-127). Les mêmes désirs opposés tourmentent d'autres autobiographes femmes, telles George Sand ou Simone de Beauvoir, et Eliane Lecarme-Tabone en explique clairement le pourquoi:

Écrire et publier son autobiographie suppose à la fois un grand intérêt pour son moi et le désir de le rendre public, deux attitudes contraires à l'effacement trop longtemps enseigné aux femmes et à leur relégation dans la sphère privée. Le geste autobiographique des femmes porte la marque de ces injonctions et de ces interdits, et révèle souvent une tension entre le désir de se dire et l'envie de se cacher (Lecarme-Tabone 2002: 58)

Contrairement à son premier texte autobiographique, dans lequel Françoise Mallet-Joris célèbre avec émerveillement son métier d'écrivain, sa véritable passion, sa vie (Mallet-Joris, 1963: 17), dans le troisième, elle le démythifie. L'auteure ne le considère que comme son "gagne-pain" grâce auquel elle peut refaire les carrelages dans sa maison ou acheter une machine à laver (Mallet-Joris, 2000: 254). Elle avoue en avoir souvent assez, "détester écrire" (Mallet-Joris, 1975: 29), devoir siéger à des comités de lecture, apparaître à la télé, donner des interviews, se faire photographier (même avec ses enfants), promouvoir ses livres pour entretenir sa famille. Pourtant, quant à celle-ci, la romancière reste très discrète.

\section{Récit des autres avant soi: La Maison de papier}

Elle en livre plus de détails dans La Maison de papier, son texte le plus traduit et le mieux vendu, que la critique qualifie de "livre enchanteur, drôle et stimulant intellectuellement" (Petit, 2001: 115). Il constitue une réponse aux questions qui lui étaient souvent posées sur sa façon de concilier la création littéraire avec des tâches de mère de famille, ainsi que sur sa conversion au christianisme. Cependant, encore une fois, au lieu de parler d'ellemême, Françoise Mallet-Joris parle des autres, notamment de ses quatre enfants, des amis, des voisins, des femmes de ménage espagnoles ou des inconnus qui passent à la maison. Comme l'observe avec justesse Monique Gosselin-Noat, avec cet ouvrage "on serait plus proche de la lettre à soi-même ou de l'autoportrait si elle ne s'intéressait ici surtout à d'autres qu'à elle-même" (Gosselin-Noat, 2018: 87). Le titre du livre, fort programmatique, exprime parfaitement la particularité de sa maison et, par métonymie, de son ouvrage: comme dans les demeures japonaises si mal fermées, où chacun peut entrer à sa guise, le foyer de cette famille est une "maison de papier, maison aux portes sans cesse battantes" où tout "se perd, tout 
fuit, tout entre" (Mallet-Joris, 1970: 74). "Des amis entrent et sortent, dînent et déjeunent. Des animaux même s'installent, apparemment de leur propre initiative" (Mallet-Joris, 1970: 72). C'est "tout qui entre", c'est justement les bribes de la vie des autres, ces particules élémentaires du simple et banal quotidien qui composent son récit. L'écrivain la présente dans toute son ostensible banalité: nous assistons aux conversations, aux petits déjeuners de famille et aux dîners d'amis, aux préparatifs des enfants à l'école, nous sommes témoins de petits incidents sans importance, des faits et gestes, de tout ce qui semble insignifiant. À titre d'exemple, citons une des conversations avec sa fille Pauline:

-Pauline, prenant son petit déjeuner:

-Tu sais, maman, tu as vraiment fait beaucoup de progrès.

- Ah oui?

-Oui. Quand j'étais petite, tu t'impatientais plus souvent, et tu étais moins gaie, tu voulais toujours faire de l'ordre. Moi, je trouve, vraiment, que tu as fait des progrès.

-Merci, ma chérie (Mallet Joris, 1970: 168).

La matière influe sur la manière, la forme du récit s'adaptant à son sujet. Françoise Mallet-Joris affirme son incapacité de parler de son quotidien autrement qu'à travers des images, ne savoir que donner un reflet des réalités en question, sans tenter de les saisir dans des formules précises et closes, de les "définir". Elle en fait un tableau, "une aquarelle" (Mallet-Joris, 2000: 169), car pour elle, "le souvenir c'est une image":

Dans le passé, les choses dont je me souviens vivement sont coupées en elles. Elles forment une série de tableaux, éclairés, mais entre ces tableaux je ne me souviens presque de rien. [...] Je crois qu'il y a une relation assez étroite entre le fait que je me souvienne, par flashes, par tableaux, vivement éclairés, [...] et le fait que je n'aime pas les transitions, que je n'aime plus du tout écrire des transitions. Par contre, écrire par tableaux très coupés correspond beaucoup plus au mécanisme naturel de mon esprit (Detry, 1976: 32-33).

Cette façon spécifique dont travaille sa mémoire influe considérablement sur la structure mosaïquée du récit. L'ouvrage constitue un ensemble hétéroclite de chapitres d'inégale longueur, coiffés d'un titre thématique (p. ex. "Isabelle, Cuba et la révolution", "le marchand de plumeaux", "le progrès", "l'argent”, etc.) et diversifiés génériquement : nous y trouvons des portraits, scènes de genre, anecdotes, dialogues, micro-essais, fragments de confessions et de méditations ou passages métatextuels. L'hétérogénéité affecte aussi les composantes poétiques de l'œuvre': l'auteure met un scène une foule de personnages de toute condition, de tout âge et tout caractère et pervertit la structure temporelle qui occasionne un va-et-vient continuel entre le passé et le présent jusqu'à en faire un pot-pourri chronologique.

6 Elle brasse aussi des catégories esthétiques normatives: l'humour, le comique, le tragique, le sublime, le kitsch, l'ironique et le pathétique. La plus dominante est l'humour qui, tantôt hilarant tantôt grinçant, procure à son récit un charme irrésistible. 
Il ne s'agit donc pas d'un "récit rétrospectif de sa propre existence", linéaire et ordonnée chronologiquement, mais de "roman-essai", de "catalogue", de "bric-à-brac", de "blocnotes" (Lubas-Bartoszyńska, 2003: 135) où elle consigne des fragments de vie des autres, morcelés et découpés en séquences thématiques, où se succèdent réflexions générales, bribes de son passé, esquisses romanesques, croquis du landernau littéraire parisien. Il faut souligner que l'écrivaine ne raconte pas, mais brosse en quelques mots des images-scènes lapidaires, sans pathos ni encombrement rhétorique, "sans but, sans problèmes, sans message" (Mallet-Joris, 1970: 204). Dans La Maison de papier, l'histoire se désagrège en images et pas en histoires. Mais ce manque de but n'est qu'apparent, la romancière sait parfaitement à quoi va mener son exploitation de menus événements de la vie quotidienne: “J'ai l'espoir qu'un but, un message, passeront malgré moi, du fait que c'est moi tout entière qui m'exprime, dans cette histoire, dans ces images qui m'enivrent un peu" (Mallet-Joris, 1970: 204). "Mais qu'est-ce que c'est l'écrivain séparé de tout ce qui l'environne, le conditionne, le nourrit ? ", se demande-t-elle à un autre endroit (Mallet-Joris, 1970: 194). Le récit de vie de ses proches, de son quotidien, est une forme fort décalée du récit de soi, car il parvient à tracer, en filigrane, le portrait de l'auteure en tant que mère de famille.

\section{Auto-bio-graphie: La Double confidence}

Ce n'est que vers le soir de sa vie que l'écrivaine réussit enfin à s'ouvrir et lever un voile sur son expérience intime ${ }^{7}$. En 2000, elle publie chez Plon La Double confidence, œuvre mi-autobiographique, mi-biographique, dans laquelle elle croise sa propre biographie avec celle de la poétesse romantique Marceline Desbordes-Valmore (1786-1859) dont la vie ressemble à bien des égards à la sienne. Ces correspondances, que Françoise Mallet-Joris découvre par-delà le temps, la poussent à s'interroger sur elle-même, sur son rapport au monde, sur son enfance et avant tout sur sa relation ravageuse avec sa mère et les causes de leur mésentente qui a duré plus d'un demi-siècle. Cependant, en parler s'avère très difficile, ce dont témoigne la forme peu habituelle de son récit autobiographique. L'écrivain ne le compose pas de manière conventionnelle, ne le concentre pas sur son trajet existentiel, mais crée une œuvre hybride qui échappe à toute classification générique univoque: c'est un habile mélange de la biographie, du récit de deuil, du récit de filiation, de la chronique de la vie littéraire parisienne de la première moitié du XIX ${ }^{\mathrm{e}}$ siècle et du portrait de sa mère, l'écrivain Suzanne Lilar. En effet, c'est la mort de celle-ci, survenue en 1994, qui pousse Françoise Mallet-Joris à se tourner vers sa personne, à revenir vers son enfance et renouer le dialogue brisé avec la mère, il y a une cinquantaine d'années. L'écrivain s'interroge elle-même sur son recul devant

7 Cette réticence devant l'énoncé autobiographique nous fait penser à Nathalie Sarraute et Marguerite Yourcenar: la première écrit Enfance (1986) à quatre-vingt-trois ans, la seconde commence sa trilogie familiale Le Labyrinthe du monde à soixante et onze ans. 
le geste autobiographique et, par là, dévoile les causes de sa réserve observée à l'égard de la confidence intime ${ }^{8}$ :

\begin{abstract}
Manque de courage? Délicatesse excessive? Car il est bien difficile souvent de départager l'exhibitionnisme de l'intrépidité. Et est-ce pudeur, goût de la fable, est-ce mon langage à moi qui passe par la fiction pour y voir plus clair, qui fait que, au cours d'un séjour en Belgique prolongé par une maladie propice, je me mets à prendre des notes et à élaborer des plans sur la Guadeloupe en 1802, alors que je pourrais, enfin, parler de la mère? (Mallet-Joris, 2000: 209).
\end{abstract}

En s'appuyant sur la vie de la poétesse romantique, tel un miroir dans lequel elle voit se refléter la sienne, Françoise Mallet-Joris se retourne sur son passé et "fait se rejoindre ses dix ans et soixante-dix" (Mallet-Joris, 2000: 12). Écrire sur l'autre et, de plus, tellement semblable, déclenche la mémoire de soi et fait remuer tout ce qui reposait en bas de son âme: il lui permet de s'observer, de se sonder, de se poser des questions qu'elle évitait, de faire resurgir le refoulé. L'écrivaine exprime de façon explicite les enjeux de son projet auto-bio-graphique:

Je veux écrire sur Marceline. Je veux m'aider d'elle pour comprendre mes longues années d'angoisse et d'efforts, mes brèves paix inexplicables, l'impossibilité pour moi, "qui ai embarqué" et ne puis plus revenir en arrière, de me situer pendant cette traversée qui pourtant touche à sa fin (Mallet-Joris, 2000: 40).

Et à un autre endroit:

Et à la lueur de cette vie de femme, de cette œuvre de femme, dans cette lumière vacillante, éternelle, de Marceline, voir un peu plus clair sur ma vésicule biliaire à moi (Mallet-Joris, 2000: 28).

Ainsi, La Double confidence s'approche du récit de filiation dans le sens où l'entend Dominique Viart. C'est le récit de l'autre - de Marceline Desbordes-Valmore - qui constitue "le détour nécessaire pour parvenir à soi, pour se comprendre dans cet héritage: le récit de filiation est un substitut de l'autobiographie" (Viart \& Vercier, 2005: 75). L'auteur considère Marceline Desbordes-Valmore comme son "amie", son "révélateur", la "pierre de touche" de sa vie d'écrivain et de femme (Mallet-Joris, 2000: 280). Non sans raison, car il y a entre elles de nombreux points communs: mêmes origines flamandes, mêmes difficultés de l'écriture, même bonheur de la maternité, mêmes erreurs de jeunesse et désespoirs de l'amour. Elles ont eu toutes les deux une vie sentimentale très passionnée, des enfants qui ont été pour elles les plus importants. Elles ont eu aussi des difficultés financières: Marceline a connu la misère, recevait une petite pension de l'État pour sa carrière littéraire, dispensait son argent à qui en

8 Notons qu'il a fallu six ans pour que le livre voie le jour. 
avait besoin; Françoise Mallet-Joris a plutôt vécu, de manière souvent précaire, de ses succès littéraires, ce qui l'obligeait à écrire tous les jours et à participer activement à la vie littéraire parisienne. Qui plus est, toutes les deux ont toujours suivi leur voie personnelle à rebours des tendances et des modes littéraires. Mais au-delà de tout, ce qui crée un rapprochement actif entre les deux écrivains, c'est les mêmes blessures de l'enfance, venues d'une mère qui n'a pas su leur montrer sa tendresse. Ce rapport problématique à la génitrice marquera toutes les relations intimes des deux femmes et fera que l'amour et la souffrance, la joie et la tristesse, seront pour elles inséparablement mêlés. Françoise Mallet-Joris avoue à ce propos:

[...] tous mes rapports, d'amour ou d'amitié, se sont plus ou moins imprégnés de cet amour maternel qui m'avait si longtemps manqué. Parce qu'il m'avait manqué, je ne pouvais pas le refuser à qui me le demandait, et, lentement, cette dépendance qui n'était pas à sens unique dégradait l'amour ou l'amitié (Mallet-Joris, 2000: 250).

Non seulement ces émotions seront inséparables, mais aussi, et peut-être avant tout, indicibles. D'où vient, à notre avis, sa difficulté d'en parler: de peur d'y revenir, d'en souffrir encore une fois, ainsi que de ne pas savoir verbaliser son expérience et ses sentiments. Les réflexions de l'écrivaine sur son projet biographique semblent confirmer notre raisonnement:

À côté de cela le foisonnement, peut-être vain, de tant d'esquisses, de plans, de projets de nouvelles, de romans, à travers lesquels parfois réapparaissent, comme la chatte Émilie ${ }^{9}$, des visages et des paroles dont j'ai peur de me souvenir sans cette métamorphose [...] Si j'arrive, non sans peine, à les évoquer autrement, c'est avec une sécheresse qui me fait, à moi-même, mal (Mallet-Joris, 2000: 103).

Et plus loin:

[...] l'amour, comme on l'entend, avec halètement et sanglots de minuit, je ne puis pas en parler. Je ne puis pas. C'est tout juste si je puis - et j'ai un peu tardé, par appréhension - raconter l'histoire d'amour de Marceline (Mallet-Joris, 2000: 107).

Les thèmes de l'enfance malheureuse et de la relation conflictuelle avec sa mère se trouvent au centre du versant autobiographique de l'ouvrage. C'est la première fois que Françoise Mallet-Joris ose les aborder. Il faut toutefois noter qu'elle ne trouve pas, sinon difficilement, les mots justes pour en parler, ce dont témoigne la façon fort biaisée de son énoncé autobiographique. Évoquons, à titre d'exemple, le fragment consacré à la photographie du jardin de sa maison familiale qui en dit long sur ce qu'a été son enfance sans vraiment en parler:

9 La chatte aimée de la petite Françoise, morte de froid, enfermée dehors probablement par sa mère. Le fantôme de l'animal hante Françoise toute sa vie et lui rappelle ses douloureuses relations avec la génitrice ( $c f$. Mallet-Joris, 2000: 99). 
C'est l'hiver. Les arbres sont nus. D'ailleurs il n'y en a que trois vraiment imposants: un vieux chêne, malade, un catalpa et un tilleul qui monte jusqu'à ma chambre. L'herbe a presque disparu, rase. Les allées dévoilent leur tracé impitoyable. Seuls demeurent les buis sombres, le houx acéré aux feuilles presque noires, les deux statues mangées de lichen et d'humidité qui représentent, je crois, Hercule combattant le lion de Némée. Je crois que j'ai gardé cette photo comme une sorte d'appel à l'aide vers mes parents auxquels je ne l'ai jamais montrée. [...] Je la trouve plus parlante que les mots. "Voilà ce qu'a été mon enfance". Puis, à un moment donné, je l'ai punaisée dans un coin d'un appartement [...] où j'ai habité. Pour quel souvenir? Délectation morose? Puis je l'ai égarée. Peut-être la retrouverai-je un jour comme réapparaissent parfois les mauvais rêves. En attendant, j'ai planté mon jardin ailleurs (Mallet-Joris, 2000: 22-23).

Le lecteur qui chercherait à connaître plus de détails sur les fractures emportées dans l'enfance par la romancière et sur les origines du mal qui ne cesse de la ronger sera déçu, car elle les passe complètement sous silence. Ainsi, son récit autobiographique rend compte des insuffisances du mode d'expression traditionnel et se greffe - par conséquent - sur des ellipses narratives. Cette "zone d'absence" ne trouve du poids sémantique que lors du processus interprétatif: constitué d'une "écriture blanche" ( $c f$. Barthes, 1953), elle suppose une forte participation du lecteur qui peut déchiffrer le contenu latent et compléter le sens en comblant les lacunes textuelles. Philippe Lejeune parle à ce propos de l'autobiographie oblique et indirecte qui fournit au lecteur des données quasi psychanalytiques, mais s'arrête presque toujours au seuil de l'interprétation (Lejeune, 1991: 65-66).

L'auteure est plus prolixe sur ses relations avec la mère, ce qui s'explique par la genèse de l'œuvre: celle-ci naît d'une perte, d'un vide laissé par la disparition de la génitrice et constitue une tentative de rétablir le lien brisé avec elle. Leur mésentente était un mal qui taraudait la romancière depuis ses treize ans, un trouble intime qui résistait à toute épreuve de représentation directe. Elle a tenté à maintes reprises de le soigner par l'écriture, en peuplant ses romans de "mauvaises mères, femmes froides et égoïstes dont l'indifférence affective à l'égard de leurs enfants amène une cascade de malheurs et entraîne des conséquences désastreuses sur leur vie" (Bizek-Tatara, 2015: 26).

Cette impossibilité de dire cette relation tendue se signale aussi en filigrane dans ses textes autobiographiques antérieurs où elle passe complètement sa mère sous silence. Elle parle de ses enfants, de ses quatre maris, de sa vie quotidienne, de son écriture, mais point de Suzanne Lilar, la grande absente. Nous savons bien que le silence est éloquent et représente une stratégie pour exprimer ce qu'on ne veut ou ne peut dire, pour communiquer l'incommunicable. Le silence de l'auteure est donc paradoxalement très parlant: il exprime l'immensité du trouble émotionnel que constitue sa mésentente avec la mère, mésentente douloureuse et, par là, indicible. D'où le recours à la vie de la poétesse romantique, embrayeur de la mémoire de soi où se trouvent déposés des souvenirs muets: cette archéologie que mène la romancière la fait revenir sur son passé, la pousse à l'inventorier et y voir plus clair. Sans ce détour, qu'elle qualifie explicitement d'“alibi”, de "paravent" (Blandiaux, 2001), elle n'aurait pas 
osé décrire sa relation à la génitrice ni lever un voile sur leur duel violent. Pour pouvoir parler d'elle-même, elle doit parler d'une autre.

Éveillé par le travail biographique, son passé surgit dans le présent et lui fait revoir divers épisodes liés à sa mère qu'elle a longuement refusés et refoulés. L'auteure évoque des situations familiales marquantes, certains comportements, gestes, paroles de Mme Lilar et essaye de les analyser, les expliquer et les comprendre. C'est ainsi qu'en investiguant sur les sources du désespoir de la mère de Marceline et de la distance creusée entre elles, Françoise Mallet-Joris se rappelle une promenade à Anvers pendant laquelle elle a vu en sa mère une autre femme qu'elle connaissait, un personnage distant et étranger, pour qui elle n'était qu'accessoire:

Je suis encore enfant [...] Ma mère me tenait par la main, et elle pleurait. [...] je croyais à l'époque, qu'elle n'avait pas de faiblesses. Je ne l'avais jamais vue pleurant. J'étais trop jeune pour qu'elle me parlât de ses soucis de femme. Et pourtant on sait, on sent: je sus qu'elle pleurait d'amour. De quel amour, je n'en sais rien. Mais la nature de son chagrin ne m'échappait pas. Qu'elle fût venue pleurer loin de sa maison, dans cet endroit désert, me désolait. Et plus encore, bien qu'elle tînt ma main très serrée dans la sienne, le sentiment de lui être d'un bien piètre réconfort. Je ne me croyais ni mal-aimée, ni malheureuse, encore moins maltraitée, mais accessoire. Nullement nécessaire. L'essentiel, dans la vie de ma mère, n'était évidemment pas d'être ma mère. Je la vis, de ce jour, comme une personne. Une invisible paire de ciseaux avait coupé en silhouettes séparées l'image l'enfant-et-sa mère, à laquelle j'avais voué ma dévotion (Mallet-Joris, 2000: 49).

De tels fragments, éparpillés çà et là dans la masse diégétique du texte, composent progressivement, comme des pièces de puzzle, le portrait de la génitrice qui parvient, au terme du livre, à corriger l'image que la fille avait d'elle et la réhabiliter en tant que mère.

Cette investigation de l'antériorité mène l'auteure à une investigation d'intériorité: elle s'interroge sur sa relation avec la mère et leurs fréquents duels, analyse leurs causes et effets. À soixante-dix ans, Françoise comprend enfin ses choix, ses décisions et les conséquences qu'ils ont eues sur leurs rapports. Évoquons, à titre d'exemple, sa première histoire d'amour. C'est pour éveiller l'attention de sa "stoïque et cruelle mère" (Mallet-Joris, 2000: 134), pour la "contrarier", "rivaliser avec elle, et même l'étonner" (Mallet-Joris, 2000: 118) que la jeune Françoise séduit l'amant de sa génitrice. Nous lisons: "Défi? Hommage? Il y avait une part d'amour, aussi, dans ce qui devint avec le temps une liaison. On aime souvent une arme, un couteau" (Mallet-Joris, 2000: 53). L'auteure admet que cet acte audacieux qui, au fond, n'était qu'un cri de détresse d'une fille rejetée, a alimenté le clivage entre elles et a eu des conséquences fâcheuses sur leurs relations: il a fait d'elles des rivales qui se livraient une guerre permanente et se blessaient mutuellement. La métaphore qui décrit la longue période de leur mésentente le confirme parfaitement: "Maman m'aima sans problèmes jusqu'à l'adolescence, puis de nouveau, très tard, quand elle est devenue en quelque sorte mon enfant : entre les deux, buissons d'épines" (Mallet-Joris, 2000: 120). 
Ce "plus tard" est le moment sur lequel la romancière s'attarde le plus et elle le décrit de façon développée. Quelques semaines avant la mort de Suzanne Lilar, elles parviennent enfin à communiquer et à se réconcilier. Pourtant, elles ne parlent pas de leurs émotions longtemps enfouies, ne s'avouent pas leur amour et douleur, leurs déceptions et haines, car la mère disparait bientôt. D'où le projet d'en faire un livre: la fille veut y revenir, ausculter l'histoire de leur mésentente et la retravailler.

Inspiré par la mort de la génitrice, ce récit autobiographique prend les allures de l'écriture du deuil. C'est un projet par excellence réparateur, à la fonction thérapeutique: il aide la fille à surmonter la perte en reconstituant l'objet perdu, la mère, et par extension, leurs rapports mutuels. Comme le note Simon Harel, "la mémoire est au cœur d'une activité qui affirme une reconquête de l'objet perdu" (Harel, 1994: 116). Ce retour en arrière, difficile et chargé d'émotivité, rend possible une meilleure compréhension de la mère, de l'auteure ellemême et de leur relation réciproque. Ainsi, comme le fait remarquer Renata Bizek-Tatara:

Le travail du deuil constitue un faire textuel, une sorte d'écriture engagée qui revisite les blessures du passé et fait revivre la relation douloureuse avec la mère défunte pour la guérir. Il privilégie la réparation symbolique de leur rapport tendu, mène vers une réconciliation intérieure chez la fille et lui sert de source de réconfort, car il rend ses souvenirs moins navrants (Bizek-Tatara, 2015: 33).

Il comporte en effet une dimension éthique dans le sens où il offre la possibilité de changer l'expérience douloureuse en sujet littéraire. "Nommer le désespoir, c'est le dépasser", écrit Albert Camus (Camus, 1951: 314), et Françoise Mallet-Joris semble poursuivre cette voie: verbaliser les événements traumatisants, c'est les revivre, les retravailler, les modifier et essayer de s'en remettre. C'est, pour emprunter les mots à Alexandre Gefen, la “possibilité d'une guérison de l'auteur et d'une salvation de la mémoire du défunt” (Gefen, 2017: 133).

La difficulté à dévoiler sa vie influe autant sur la structure binaire de l'œuvre - ce que son titre reflète à merveille - que sur le choix des stratégies discursives. Le récit autobiographique, composé de fragments épars, morcelle la trame narrative du récit biographique consacré à Marceline Desbordes-Valmore. Constitué de séquences de longueur variée, allant le plus souvent de trois, quatre phrases à des paragraphes plus composés, le récit autobiographique apparaît moins continu, moins ordonné et moins substantiel que le récit biographique. Toutefois, à force d'être "réglementé", il devient un bien rare et, par là, chaque son apparition est frappante. Ces "îlots narratifs autobiographiques qui parsèment, par intermittence, la biographie de la poétesse romantique, interpellent le lecteur et le poussent à se poser la question sur les raisons de cette disproportion flagrante qui travaille la structure interne de La Double confidence" (Bizek-Tatara, 2015: 31).

Constitué de bribes, de non-dits, de silences et d'ellipses narratives, le récit autobiographique ne trouve son poids sémantique qu'à travers la lecture du récit biographique, indispensable pour sa compréhension: il l'alimente, le complète, comble les blancs et, ainsi, lui 
donne sens. Bien que les deux récits alternent, ils n'ont pas le même statut. Si l'histoire de la vie de la poétesse peut se lire séparément sans détruire sa logique interne, celle de Françoise Mallet-Joris ne le peut pas, car elle est trop lacunaire et morcelée. En effet, ce récit de soi ne se déploie pas selon une linéarité chronologique restituée, mais au gré des souvenirs de l'auteure qu'éveille le travail biographique, ce qui entraine une construction fragmentée et un désordre chronologique. L'écrivain ne nous donne pas de développement cohérent de son vécu: chaque séquence autobiographique rapporte un épisode isolé qui ne prend sens que lorsqu'il est précédé de la lecture d'une séquence biographique antérieure. Cette "insuffisance" qu'affiche le récit autobiographique traduit, à son tour, la difficulté de l'auteure à parler de son expérience intime.

Ajoutons, avant de conclure, que la romancière alimente aussi ses textes de fiction de son vécu. Les deux premiers romans, Le Rempart des béguines (1951) et La Chambre rouge (1955) font écho à la séduction de l'amant de sa mère par la jeune Françoise: dans le premier, l'hérö̈ne entretient avec sa marâtre une relation lesbienne très perverse, dans le second, la mère et la fille partagent le même amant et deviennent rivales. La biographie d'une mystique, Jeanne Guyon (1978), et le roman Divine (1991) s'inspirent par contre de la conversion de l'écrivaine belge au christianisme. Mais c'est le motif de la relation tendue et conflictuelle entre fille et mère qui est le plus récurrent. Les romans qui l'explorent sont légion. Dans Adriana Sposa (1990), l'héroïne éponyme abandonne à jamais sa fille de trois ans et fuit en Italie pour y vivre avec son amant. Il en est de même dans Divine (1991) où Gisèle laisse sa fille Jeanne à l'orphelinat et continue à mener une vie frivole. L'enfant n'est pour elle "qu'un accident bénin, pas plus grave qu'une rougeole. Un accident du corps, sans plus" (Mallet-Joris, 1991: 32). Dans La Tristesse du cerf volant (1986), les frères Chris et Clara Metthyssen sont hantés par l'absence d'amour maternel qu'ils comblent en nouant une relation incestueuse. Qui plus est, ce désamour maternel se transmet de génération en génération: Clara, n'étant pas aimée par sa mère, n'aime pas sa fille Marianne qui, à son tour, n'éprouve rien pour sa fille Nadine. La relation entre la mère et la fille qui est au cœur de Sept démons dans la ville (1999) n'est pas plus reluisante: Alix Desroches et sa fille Evelyne rivalisent pour capter l'affection de Maurice Desroches - leur mari et père, en s'accusant réciproquement d'être la cause de son départ et en s'en punissant cruellement. Elles s'aiment mais étant toutes les deux trop orgueilleuses, trop endurcies par une émulation continuelle, elles sont incapables de se parler franchement et de s'avouer leurs sentiments. Enfin, le roman Ni vous sans moi, ni moi sans vous (2007) porte sur la recherche de la mère et sur la question d'une filiation manquée. Cette récurrence continuelle de la figure de mère mauvaise ou absente, ainsi que du thème de la relation mère/fille conflictuelle n'est pas uniquement un choix thématique de l'écrivain: elle constitue aussi une projection et une transcription, chaque fois remodelées, de sa relation à la mère, Suzanne Lilar. Faire de son expérience personnelle un sujet de roman est pour l'auteure une façon, fort biaisée, de parler d'elle-même, de verbaliser ses joies, ses hantises et ses tourments, bref, de se dévoiler tout en se voilant. 


\section{Conclusion}

L'impossibilité d'exprimer ses sentiments et de faire de soi-même à la fois le sujet et l'objet de l'écriture amène Françoise Mallet-Joris à préférer le fragmentaire et l'ellipse qui se prêtent parfaitement à dire des questions épineuses, douloureuses, difficiles à verbaliser, car le fragmentaire "se trouve spontanément associé à l'idée d'une défaillance, d'une faiblesse, voire d'une véritable pathologie de l'être" (Susini-Anastopoulos, 1997: 60). Quelle meilleure façon de dire cette incapacité du langage que de l'inscrire littéralement dans le texte, incapacité incarnée tant par l'inachèvement dans le discontinu du fragment que par les lacunes qui parsèment le texte. Comme le note Ginette Michaud, "le fragment exhibe en lui-même [...] ce manque du langage" (Michaud, 1989: 36) et traduit la carence de mots aptes à énoncer une expérience difficile à assumer. L'architecture mosaïquée des récits autobiographiques et le style de la romancière, qui procède par bonds, flashes, phrases courtes, coupées et lapidaires, traduisent parfaitement ce malaise intérieur.

Cette difficulté à $s$ 'autotextualiser - si on nous permet ce néologisme - influe aussi sur le contenu de ses textes autobiographiques et, par conséquent, sur leur identité générique. Pour parler d'elle-même ou dévoiler, même indirectement, quelques détails de son vécu, l'auteure doit, au préalable, passer par l'épreuve d' “ils", par l'écriture de l'autre, ce qui lui permet de la ramener à elle-même. Dans les récits de soi de Françoise Mallet-Joris, ce sont les autres, c'est leur vécu qui la fait sortir de sa coquille et déclenche son acte incertain et hésitant d'écriture du moi. Il en résulte des textes génériquement hybrides, impurs et indécis, jouant avec les frontières de l'autobiographie jusqu'à les dépasser et les reconfigurer.

\section{Références bibliographiques}

Barthes, Roland. 1953. Le Degré zéro de l'écriture. Paris, Seuil.

BizeK-TATARA, Renata. 2015. "La communication problématique entre la mère et la fille. La vie et l'œuvre de Françoise Mallet-Joris" in Synergies Pologne, n 12, 25-36.

Blandiaux, Irène. 2001. "Un miroir, deux reflets" in $D H$. Be, le 19 janvier: < http://www.dhnet.be/medias/livresbd/un-miroir-deux-reflets 51b7d913e4b0de6db9918e7e > [10/04/2019].

Camus, Albert. 1951. L’Homme révolté. Paris, Gallimard.

Detry, Monique. 1976. Françoise Mallet-Joris: dossier critique et inédits. Paris, Grasset.

Ernaux, Annie. 2000. La Vie extérieure. Paris, Gallimard, coll. "Folio".

GEFEn, Alexandre. 2017. Réparer le monde. La littérature française face au XXIe siècle. Paris, Ed. Corti. 
Difficulté de Se Dire. Sur L'ÉCriture autobiographique de Françoise Mallet-Joris

Gosselin-Noat, Monique. 2018. "Note sur La Maison de papier de Françoise Mallet-Joris" in Société de Littérature du Nord, nº 71, 87-95.

Harel, Simon. 1994. L'Écriture réparatrice. Le défaut autobiographique: Leiris, Crevel, Artaud. Montréal, XYZ.

LeCarme Jacques \& LeCarme-Tabone Eliane. 1997. L'Autobiographie. Paris, Armand Colin.

LeCARme-Tabone Eliane. 2002. "XX in Les Écritures du moi. De l'autobiographie à l'autofiction. Le magazine littéraire, $\mathrm{n}^{\circ} 409$, 56-59.

LeJeune, Philippe. 1971. L'Autobiographie en France. Paris, Seuil.

LeJeune, Philippe. 1991. La Mémoire et l'oblique. Georges Perec autobiographie. Paris, P.O.L.

LeJeune, Philippe. 1975. Le Pacte autobiographique. Paris, Seuil.

Lubas-BartoszyŃSKa, Regina. 2003. Powieść kobieca wobec powieści współczesnej. Przykład Françoise Mallet-Joris in Pisanie autobiograficzne wkontekstach europejskich. Katowice, Śląsk Wyd. naukowe, 132-141.

Mallet-Joris, Françoise. 1963. Lettre à moi-même. Paris, Julliard.

Mallet-Joris, Françoise. 1970. La Maison de papier. Paris, Grasset.

MALlet-JoRis, Françoise. 1975. J'aurais voulu jouer de l'accordéon. Paris, Julliard.

Mallet-Joris, Françoise. 1991. Divine. Paris, Flammarion.

Mallet-Joris, Françoise. 2000. La Double confidence. Paris, Plon.

Michaud, Ginette. 1989. Lire le fragment: transfert et théorie de la lecture chez R. Barthes. La Sasse, Hurtubise.

Paque, Jeannine. 2017. "Françoise Mallet-Joris, joyeusement polyvalente" in Le Carnet et les instants, $\mathrm{n}^{\circ} 193,32-36$.

Petit, Susan. 2001. Femme de papier. Françoise Mallet-Joris et son cuvre. Paris, Grasset.

Pychowska, Joanna. 2007. "Le 'Je', les histoires et le miroir humain” in Synergies Pologne, $\mathrm{n}^{\circ} 4,65-70$.

RaChWALSKa von ReJChWALD, Jolanta. 2007. "Les intermittences du je' dans La vie extérieure d'Annie Ernaux" in Synergies Pologne, No 4, 71-78.

Susini-Anastopoulos, Françoise. 1997. L’Écriture fragmentaire. Définitions et enjeux. Paris, PUF.

Viart, Dominique \& Vercier, Bruno. 2005. La Littérature française au présent, Héritage, modernité, mutations. Paris, Bordas. 